\title{
Deployment of Effective Testing Methodology in Automotive Software Development
}

\author{
P. Sivakumar ${ }^{*}$, B. Vinod1', R. S. Sandhya Devi², R. Divya1 \\ ${ }^{1}$ Department of Electrical and Electronics Engineering, PSG College of Technology, Coimbatore, India \\ ${ }^{2}$ Department of Electrical and Electronics Engineering, Kumaraguru College of Technology, Coimbatore, India \\ Email: "psk@eee.psgtech.ac.in, bvin@rae.psgtech.ac.in, sandhyadevi.rs.eee@kct.ac.in, srdivya6@gmail.com
}

Received 2 April 2016; accepted 20 April 2016; published 27 July 2016

Copyright (C) 2016 by authors and Scientific Research Publishing Inc.

This work is licensed under the Creative Commons Attribution International License (CC BY). http://creativecommons.org/licenses/by/4.0/

c) (i) 0 pen Access

\begin{abstract}
Software development in automotive industry has bestowed greater comforts and conveniences to mankind. A remarkable progress in this field often faces a setback due to minor defects in the software. So there is recurring need for standardization and implementation of testing strategies. But the process of creation of test scripts to check if the software created complies with its specifications and requirements is both time- and resource-consuming. Generating a short but effective test suite usually requires a lot of manual work and expert knowledge. Patronizing research work in this field is the need of the hour. This paper solves the problem by using Model-Based Testing where test harness and evaluation are performed economically through automation. Simulink Design Verifier and Reactis are the tools used to carry out this purpose in Adaptive Front Light System. The resulting outputs obtained from Simulink Design Verifier and Reactis using Model-Based Testing prove that short test suites can be generated for the model where full model coverage can be achieved easily through automation. The outputs of these test cases when compared with the expected outputs confirm that the model developed is working as per the requirements.
\end{abstract}

\section{Keywords}

Model Advisor, Model-Based Testing, Reactis, Simulink Design Verifier

\section{Introduction}

In this modern era, automotive industries are investing their money, energy and technological talent into the

${ }^{*}$ Corresponding author.

How to cite this paper: Sivakumar, P., Vinod, B., Sandhya Devi, R.S. and Divya, R. (2016) Deployment of Effective Testing Methodology in Automotive Software Development. Circuits and Systems, 7, 2568-2577.

http://dx.doi.org/10.4236/cs.2016.79222 
quest to develop the best software solutions that will give transport an edge. Government also puts pressure on automobile industries to reduce congestion and pollution and to follow the safety standards. As automation technology unfolds its wings, the manufacturers have to innovate and update their software. Even a small error in the software developed may lead to accidents which may be fatal. Several cars of different manufacturers have been globally recalled due to faulty airbags and faulty software that can briefly shut down the engine. Never before there were so many recalls and fixes that are directly the result of software issues. So the manufacturers recognize the need for an adaptable and comprehensive software quality strategy for the automotive software development. This is only achievable through a truly integrated testing ecosystem that focuses on risks, with complete transparency of all testing-related activities. Failures can be prevented only when a system is tested before it is marketed. In the software testing process a new technique called Model-Based Testing has become quite popular in the recent years. Model-Based Testing is an art of improving the development process by cutting down the time consumption and costs during the development of an automotive embedded system [1]. Software testing is the process of using relevant test cases from an infinite test case domain to verify the functioning of a program. Exhaustive testing is not possible or practical for most real programs. So a small number of tests must be chosen which can execute in the available time. The behavior of the system must be checked after each test case execution to determine whether there is any failure or not. This is called the oracle problem. Often manual inspection is used to check whether the outputs obtained are the same as the expected outputs in the oracle problem. But the inspection must be automated for an efficient and repeatable testing. Model-Based Testing helps in automatically selecting the test inputs as well as the generation of oracles.

In this paper, Model-Based Testing is performed using the following tools-Simulink Design Verifier, Reactis and SystemTest. By using these tools the requirements for the model are checked, model coverage is performed, and the test analysis is done. By doing this process using Model-Based Testing, the model is verified to be without any errors. Thus the errors if present in the design can be easily identified using this method during the early development stage of automotive software which reduces the total cost of testing.

\section{Model-Based Testing}

The fixing of a defect found in the design stage itself costs much less when compared to the fixing up of defects found in the final testing process before production [2]. If the fault is detected earlier, valuable time and money can be saved. To cope up with the rapid advancement in technology and to meet the higher quality needs, an effective testing methodology namely Model-Based Testing has emerged. Model-Based Design mainly deals with the creation of a system design model and use the model created for the early testing process and also for code generation from the model. Generation of test cases from the system design model automatically is one of the best features of Model-Based Testing [3]. By combining Model-Based Design and Model-Based Testing the verification of an embedded system can start early during the development, since no hardware is required. Model-Based Testing is one of the testing methodologies that automate test suites to a larger extent for a suitable model by producing test cases that can be converted into executable test scripts. Simulink Design Verifier (SLDV) which is meant for finding design errors and formal verification [4] and a test vector generator tool, Reactis Tester [5] has been used in the test flow of Adaptive Front Light System. To check the consistency of the model and whether the desired behavior has been achieved, a set of concrete test scripts is generated and the test execution tools are used to manage the tests, executed regularly and the results are recorded and analyzed [6]. Corrective action is taken if required. If any failure is observed, the source of the fault must be identified and the corrective measures can be done at the same stage itself.

\section{Testing Tools}

Simulink Design Verifier (SLDV) and Reactis are the tools used in the Model-Based Testing procedure. Simulink Design Verifier is a tool that is capable of automatic test case generation and for proving behavioral properties. An important feature in SLDV is the detection of overflows, division by zero errors as well as dead code present in the system during the design error detection process. SLDV is also used to automate requirement tracing. Thus verification and validation techniques can be used in the earlier developing stage itself. Reactis is an automatic test generation tool which can be used to test the Simulink models and to generate test cases which aims maximum model coverage. In Reactis, when an error occurs it is easier to trace an error back to its original source by backward stepping. SystemTest is a software tool that helps in the analysis of test results. The simula- 
tion outputs can be checked with the expected outputs to check for any deviations present. These results can be stored in the form of html document so that the tester can use this information for further testing.

\section{Adaptive Front Light System}

The Adaptive Front Light System as shown in Figure 1 has the ability to sense when a vehicle's headlights should be turned on and off. This system controls the low beam and high beam based on the distance of the obstacles present on the path and using the street lamp detector. The high beam is definitely off when the street lamp is present since it is not necessary to use high beam in a well illuminated road. When there is no street light, high beam and low beam will be working according to the distance signal. The light dimming control system will be triggered if the barrier detected is within $5 \mathrm{~m}$ away. If an obstacle is over $5 \mathrm{~m}$ and within $50 \mathrm{~m}$, the low beam angle adjustment will be on, and the headlamp angle will be adjusted according to the actual distance. When there are no obstacles detected within $50 \mathrm{~m}$ high beam and low beam will be at on state simultaneously. The stateflow for Adaptive Front Light System is shown in Figure 2 where transitions between different states are shown.

Model Advisor checks for the requirements for MISRA-C [7] and ISO-26262 which are the standards for safety critical automotive electrical and electronic systems. It checks for blocks that are not recommended for production code generation as mentioned by MISRA-C. It also checks the state machine produced by Stateflow charts as mentioned by ISO-26262 standard. The model developed for Adaptive Front Light System successfully passed all the above mentioned tests. Thus a model for Adaptive Front Light System has been developed and tested with Model Advisor at model level which is found to be error free.

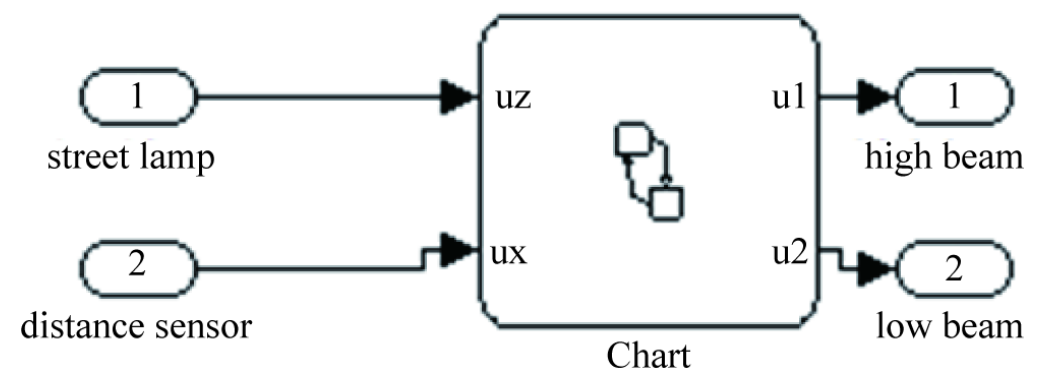

Figure 1. Adaptive front light system.

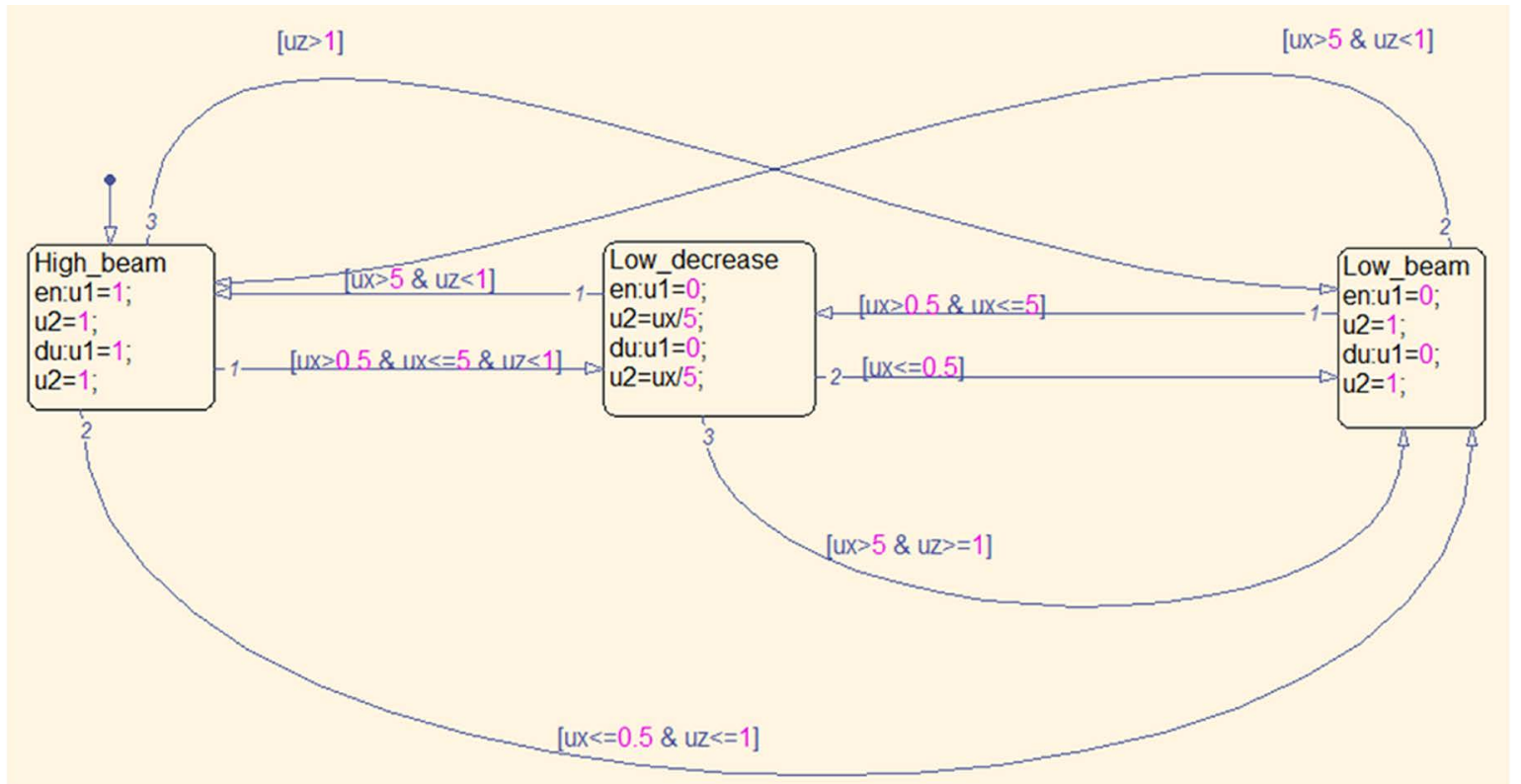

Figure 2. Stateflow of adaptive front light system. 


\section{Design Verification}

To test the system completely, first model coverage must be performed [8] on the system for which a test case has been generated. The first step in testing is test case generation which is shown in Figure 3 and Figure 4.

This test case has resulted in the decision coverage of $32 \%$ and condition coverage of $19 \%$ for the model developed where decision coverage is a metric which measures the decisions paths covered in the model and condition coverage is a metric which measures the transitions covered in the model [9]. Thus the test case generated has covered only part of the model during the test. In order to have full decision coverage the next step in testing which is the test harness must be performed. SLDV is used to automatically generate the test harness for the model developed. In the harness generated, the signal builder block containing the test cases and a test unit which is the model to be tested are present. To create the test harness for the model developed the following command is used.

>>slvnvmakeharness ('dssensorafls')

The test harness is developed as shown in Figure 5.

To save the already generated test case and to use it in the further testing process the following command is used.

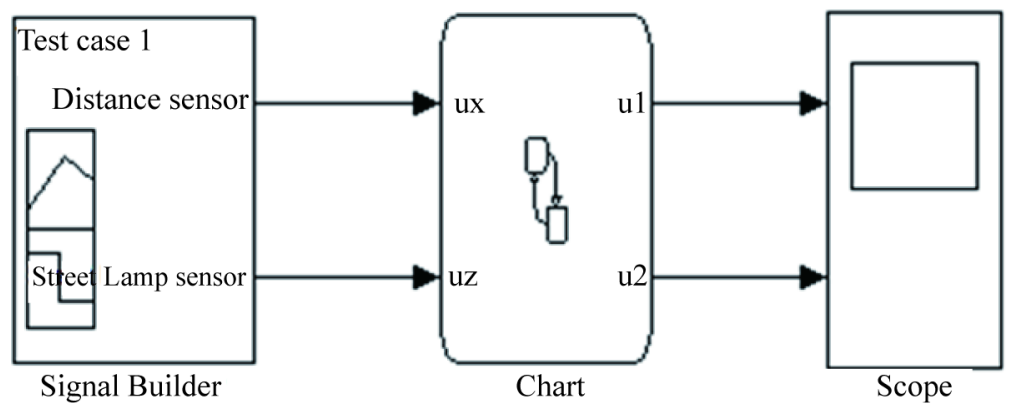

Figure 3. Test case generation for AFLS.

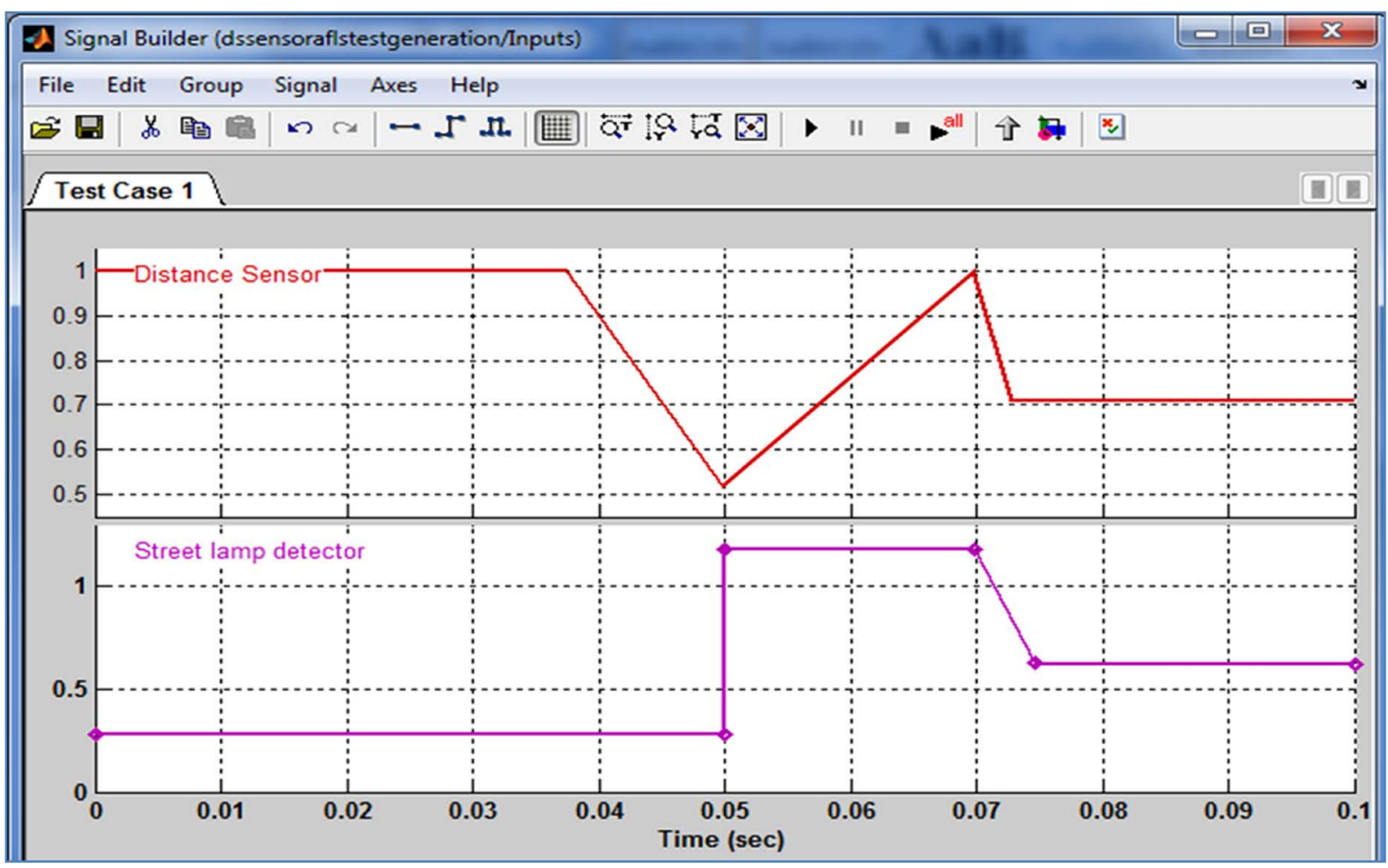

Figure 4. Test case seen in the signal builder block. 
>>dssensoraflslog1 = sldvlogsignals ('dssensorafls_harness')

>>save existingtestcaseforafls. mat dssensoraflslog1

\subsection{Combined Harness Using Simulink Design Verifier}

Thus an extended harness can be created for the model during the test harness step where the extra test cases generated help to increase the decision coverage of the model as seen in Figure 6. So that these test cases can be used during test analysis. For a test suite of a state chart to achieve full decision coverage all entry and exit paths through a state must be evaluated by test cases.

The model is tested in Design Error Detection mode to check whether there are any errors like division by zero or integer overflow errors present in the model developed and it took $17 \mathrm{~s}$ to do this analysis and no errors were found. When the Simulink Design Verifier is used in test generation mode, the transitions, conditions and state coverage are performed. The analysis time taken for this purpose is $11 \mathrm{~s}$. Only one objective is proven unsatisfiable for this model out of 47 objectives and it is shown in Table 1.

The objective proven unsatisfiable may be due to the following reasons.

1) This often indicates the presence of dead-code in the model.

2) Other possible reasons can be inactive blocks in the model.

3) In rare cases, the approximations performed by Simulink Design Verifier can make objectives impossible to achieve.

Using the test cases generated from the test harness, model coverage is performed. Since all the decision paths have been covered for the model during this analysis $100 \%$ decision coverage is obtained. Condition coverage of 96\% and Modified Condition/Decision Coverage of 92\% is obtained. Each transition or input must be changed separately while keeping the remaining inputs fixed to achieve full Modified Condition/Decision Coverage. Thus the coverage report indicates how the behavior of the model can be exercised using the generated test cases. The statistical quality of the generated test suite and the parts of the model that were not tested properly can be identified. If a particular path through the model has no test generated for it, some of the test generation parameters can be changed and the test generation step can be repeated. This is where it can be useful to use an animation tool to investigate the behavior of the path in the model and decide whether the lack of tests is due to an error in the model, a normal feature of the model, or inadequate test generation. So to improve the coverage, an abstract test can be added for this path manually or give hints to the tool to get the desired results. This test harness coverage can also be done using another tool called Reactis.

\subsection{Design Validation Using Reactis}

In Reactis automatic test case generation and the coverage analysis can be performed [10]. In order to limit the values that should be considered for the input, it must be specified in the sub range of base type in the type for port dialog box. Five tests are performed on the model with a total of 27 steps in them and the test suite generated has a decision coverage of 100\%, 97\% condition coverage and 93\% MCDC coverage for the model developed as seen in Figure 7.

The window as seen in Figure 8 shows that there is no unreachable code in the model but there are two uncovered codes.

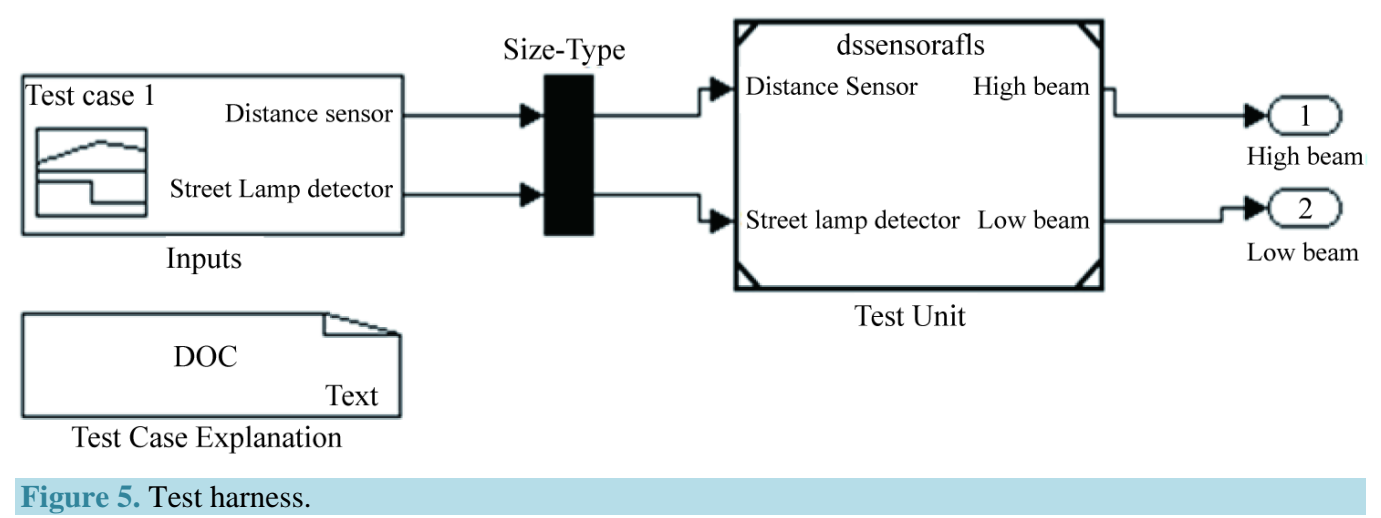




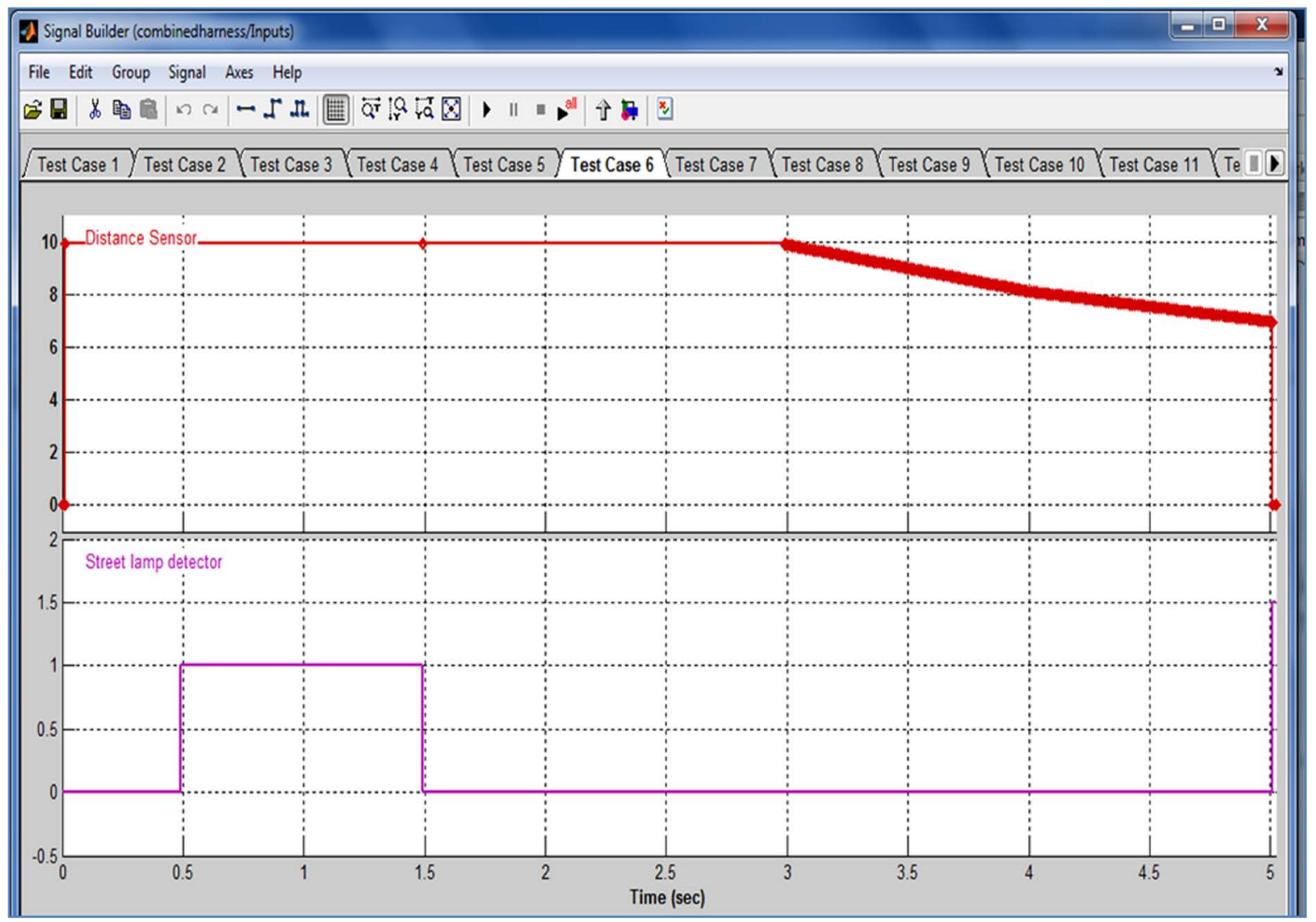

Figure 6. Extended test cases.

Table 1. Objective proven unsatisfiable.

\begin{tabular}{ccccc}
\hline$\#$ & Type & Model Item & Description & Test Case \\
\hline 43 & Condition & Chart. “[ux $>5 \& \mathrm{uz} \geq 1]$ ” & Transition: Condition 2, “uz $\geq 1$ ” F & n/a \\
\hline
\end{tabular}

Reactis has generated five test cases where the first test case contains a single sample point input, the second test case has 3 sample points input, the third test case contains 7 sample points and the remaining two test cases contain 8 sample points. All these test cases produce correct results. When the tests developed using Reactis is made to run in Simulink, there is no error when interpolation is turned off and there will be some differences in the outputs generated in Simulink when compared to Reactis outputs when interpolation is turned on because in this case Simulink will sample inputs slightly differently than the original Reactis inputs which are shown in Figure 9 and Figure 10.

\section{Test Analysis Using SystemTest}

The SystemTest software provides a framework that integrates software, hardware, simulation, and other types of testing in a single environment [11]. Predefined elements are used to build test sections that simplify the development and maintenance of standard test routines. Tests can be shared and saved throughout a development project to ensure standard and repeatable test verification. The SystemTest software offers integrated data management and analysis capabilities for creating and executing tests, and saving test results to facilitate continuous testing across the development process. The SystemTest software automates testing in MATLAB and Simulink products. The SystemTest software offers graphical test editing and repeatable test execution. The SystemTest software allows viewing the results using a workspace variable called ST results. It provides access to the test results object, which is useful for comparing the results of separate test runs and for post-processing 


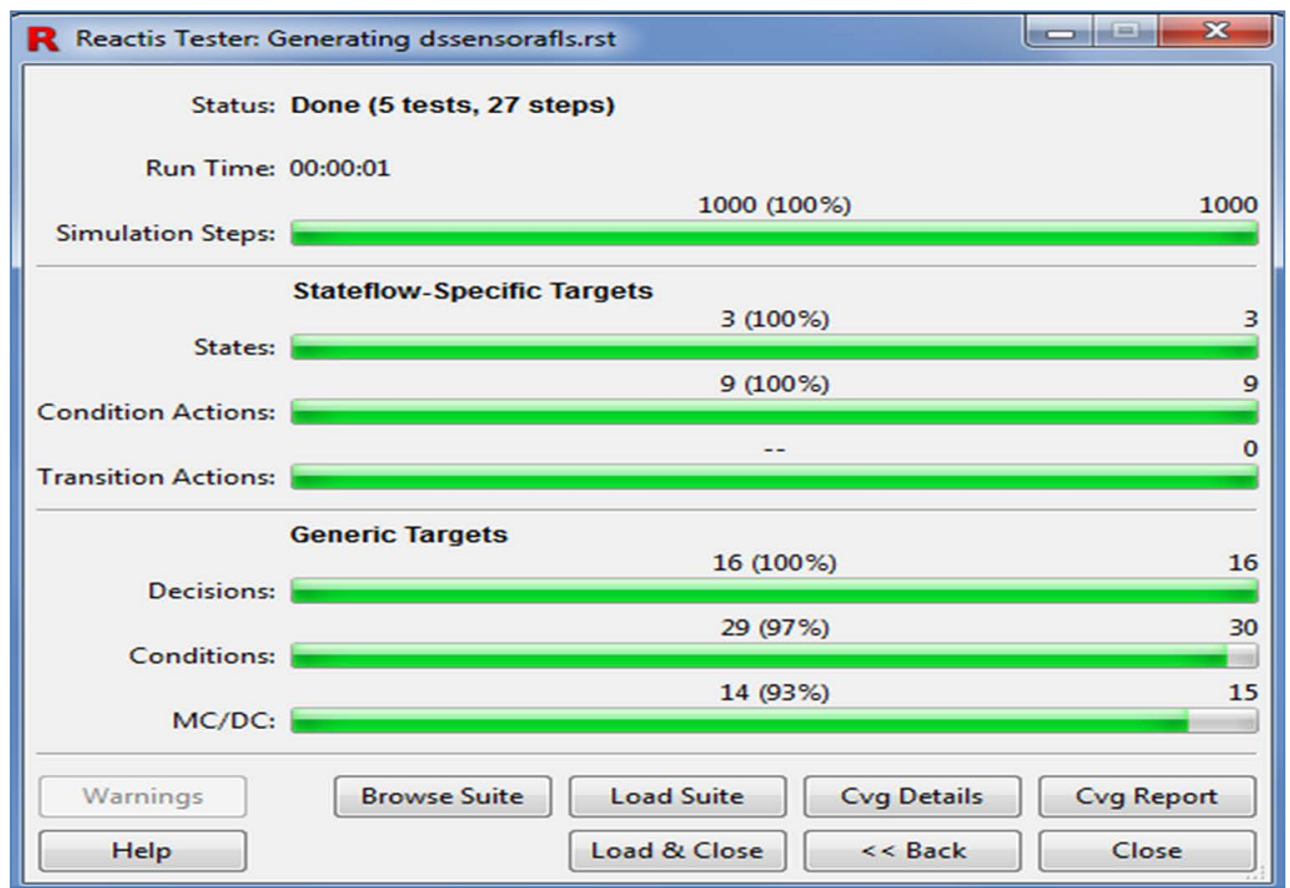

Figure 7. Test harness using Reactis.

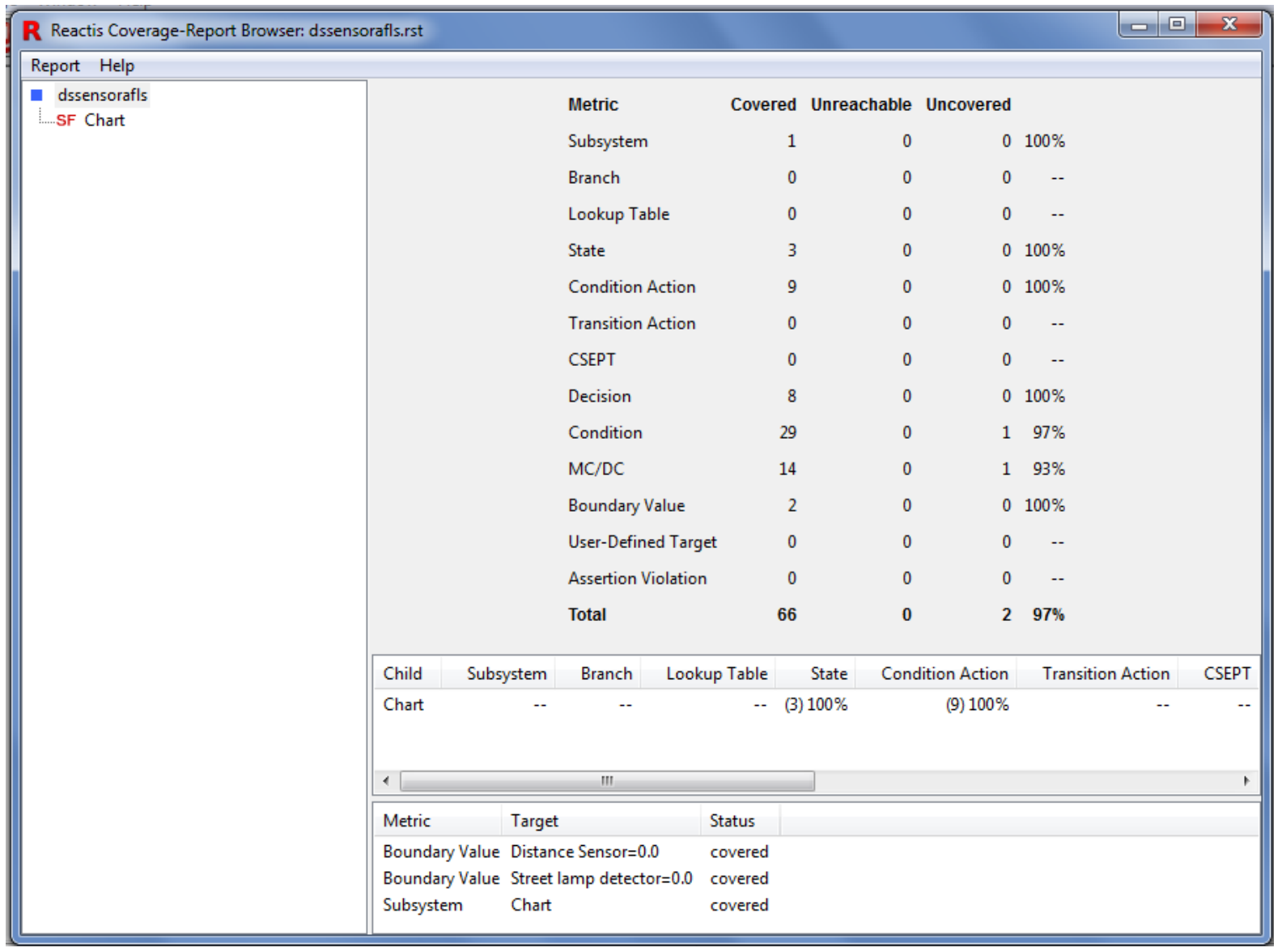

Figure 8. Coverage analysis using Reactis. 


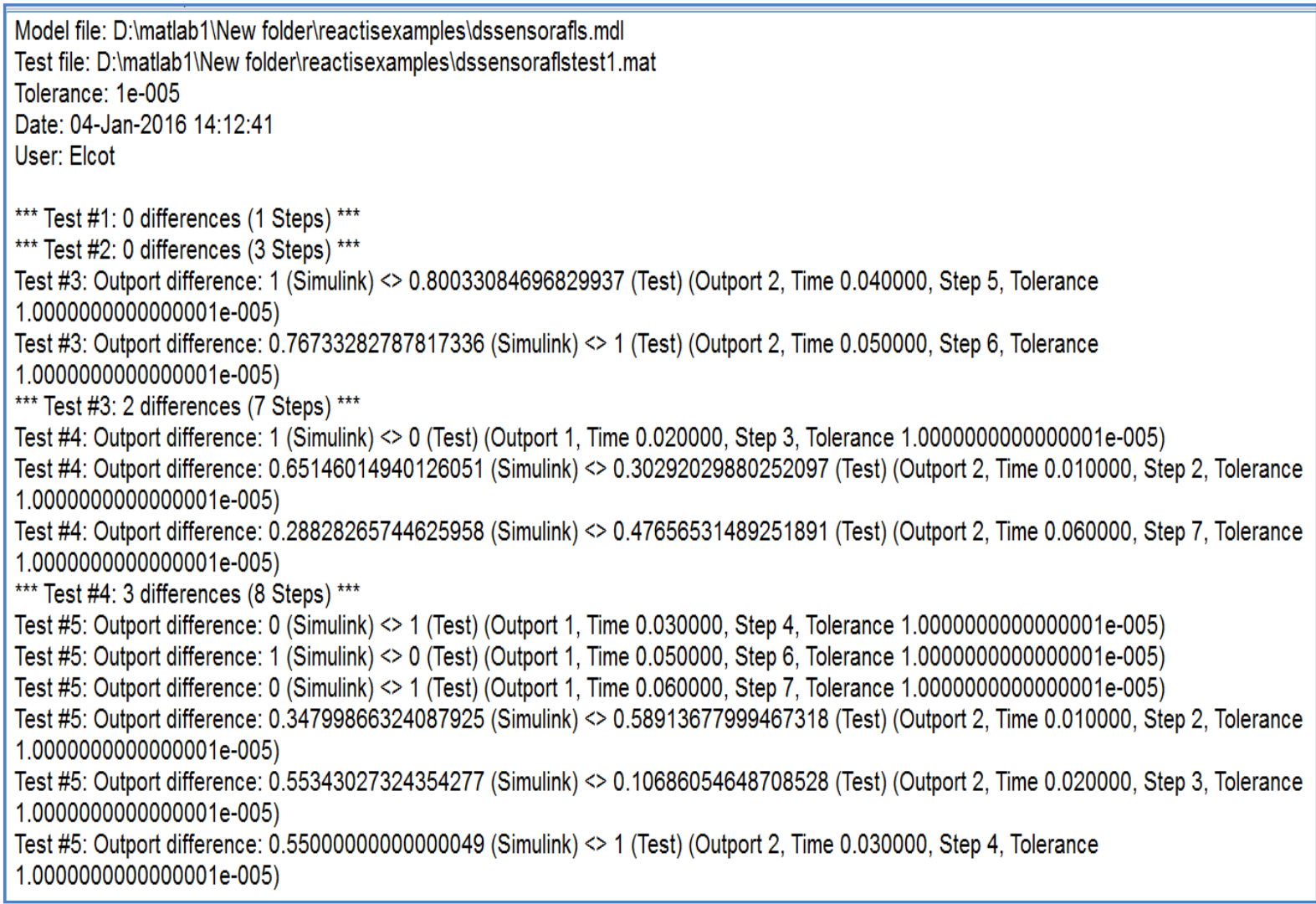

Figure 9. Differences in output from Reactis and Simulink when interpolation is turned on.

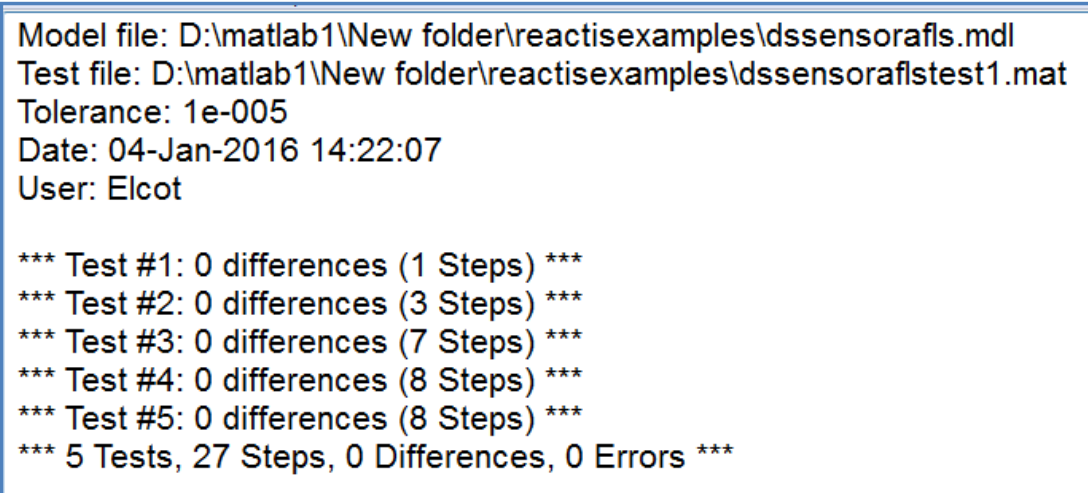

Figure 10. Differences in output from Reactis and Simulink when interpolation is turned off.

test results. In SystemTest the final process of test evaluation is performed where the test outputs will be compared with the expected outputs. During iteration 1 the input test vector is $[0.0190 .3 ; 0.0250 .2 ; 0.0310 .6]$ and for iteration $2[10 \times 3]$ vector is taken as input and their results are seen in Figure 11 and Figure 12 . The outputs generated in the SystemTest are same as the expected outputs from the model developed.

\section{Conclusion}

Manual work has been pushed to the background and day by day software is taking complete control in automotive industry. Model-Based Testing will be a blessing to rectify the errors in the software with less time consumption and yield products of high quality. This paper deals with the identification and removal of faults in automotive software development using Simulink Design Verifier and Reactis. Using these tools 100\% decision 


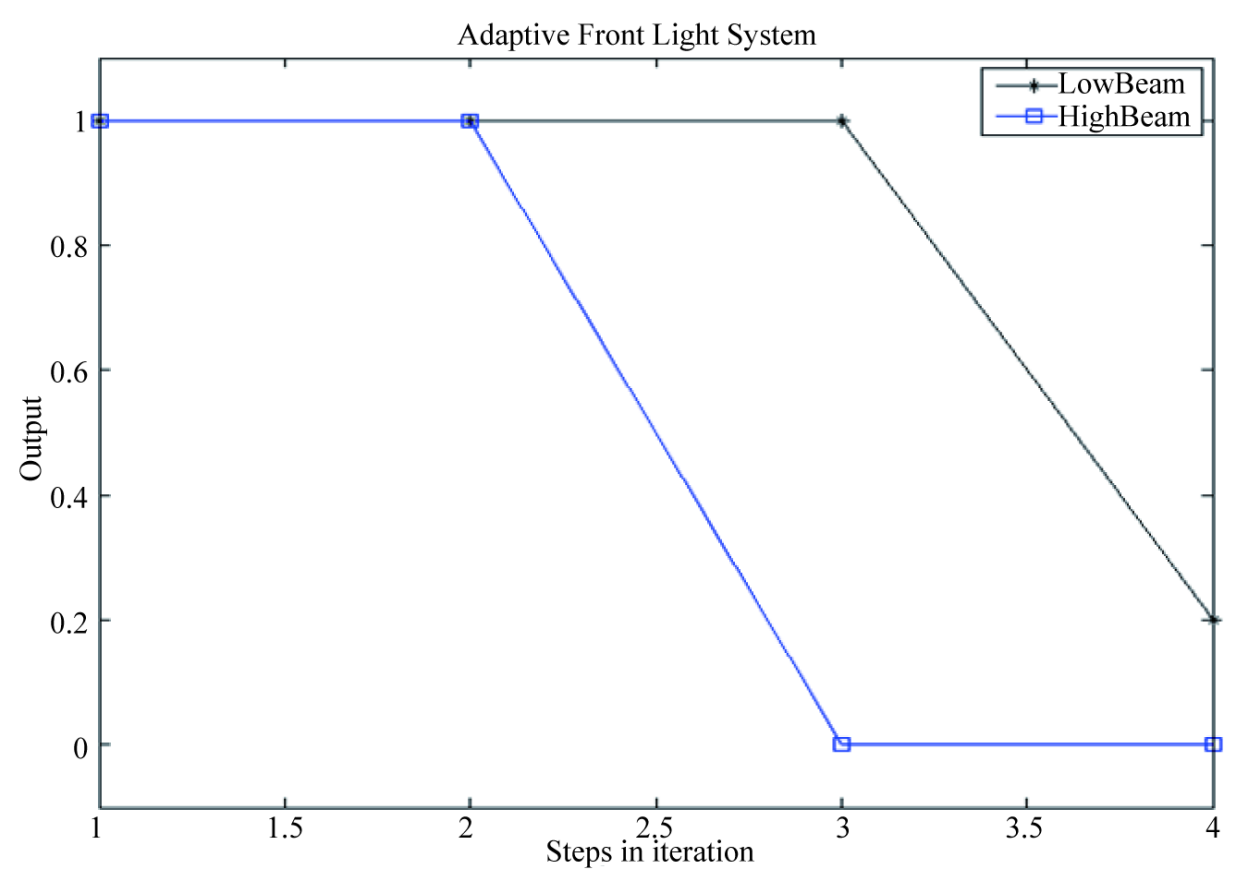

Figure 11. Iteration 1 in SystemTest.

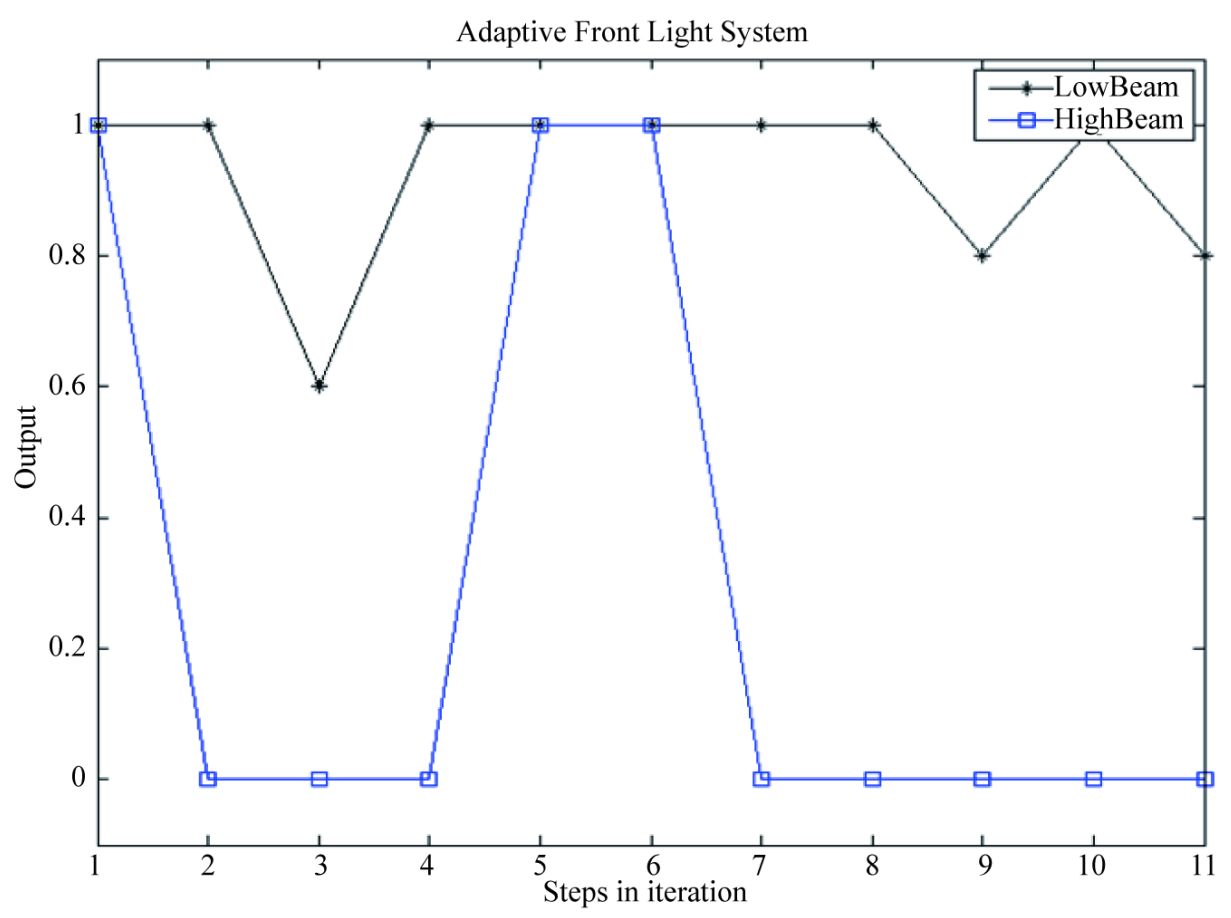

Figure 12. Iteration 2 in SystemTest.

coverage is achieved by the developed model. Reactis shows a slight improvement when compared to Simulink Design Verifier with condition coverage of $97 \%$ and modified condition/decision coverage of 93\%. Additional test cases can be added to the test suite by adjusting some parameters in the tools used to achieve full condition coverage and MC/DC coverage. SystemTest has been used to display the outputs obtained from Adaptive Front Light System in the form of graphs during the final test analysis procedure in Model-Based Testing. Thus the proposed method helps in doing the testing process at a faster pace. Today, software is being developed to regu- 
late the speed of the vehicles upon seeing the unexpected obstacles while driving up or down in a mountainous terrain. Testing methods combined with Model-Based Testing are to be developed for this type of software which will be of an important value for a safer ride and upgrading the testing skills is needed.

\section{References}

[1] Pawel, S. and Gabriel, B. (2014) Model-Based Real-Time Testing of Embedded Automotive Systems. SAE International Journal, Passenger Cars-Electronic and Electrical Systems, 7, 337-344. http://dx.doi.org/10.4271/2014-01-0188

[2] Toshiaki, K. and Masato, S. (2008) Technical Trends and Challenges of Software Testing. Science \& Technology Trends, Quarterly Review, No. 29, October 2008, 34-45.

[3] Stürmer, I., Dziobek, C. and Pohlheim, H. (2008) Modeling Guidelines and Model Analysis Tools in Embedded Automotive Software Development. Dagstuhl-Workshop MBEES, Modellbasierte Entwicklung eingebetteter Systeme IV, Schloss Dagstuhl, Germany, 7-9 April 2008.

[4] The MathWorks, Inc. (2015) Simulink Verification and Validation-User's Guide, Version 2015b. The MathWorks, Inc., Natick, Massachusetts.

[5] Reactive Systems, Inc. (2013) Testing and Validation of Simulink Models with Reactis. Reactive Systems, Inc., Cary, NC.

[6] Miller, M.D. and Ulaszek, R.R. (2012) Model-based Testing Using Branches Decisions and Options. US Patent No. 8225288.

[7] MISRA-C Guidelines (2007). http://www.misra.org.uk

[8] Justyna, Z., Ina, S. and Pieter, J.M. (2012) Model-Based Testing for Embedded Systems. CRC Press, Boca Raton.

[9] Brett, M., Amory, W. and Jon, F. (2008) Best Practices for Verification, Validation, and Test in Model-Based Design. The MathWorks, Inc., Natick, Massachusetts.

[10] Reactive Systems, Inc. (2015) Reactis-User Guide, Version 2015. Reactive Systems, Inc., Cary, NC.

[11] The MathWorks, Inc. (2010) System Test User Guide, Version 2010a. The MathWorks, Inc., Natick, Massachusetts.

\section{Submit or recommend next manuscript to SCIRP and we will provide best service for you:}

Accepting pre-submission inquiries through Email, Facebook, LinkedIn, Twitter, etc.

A wide selection of journals (inclusive of 9 subjects, more than 200 journals)

Providing 24-hour high-quality service

User-friendly online submission system

Fair and swift peer-review system

Efficient typesetting and proofreading procedure

Display of the result of downloads and visits, as well as the number of cited articles

Maximum dissemination of your research work

Submit your manuscript at: http://papersubmission.scirp.org/ 